Journal of Computer Science 3 (5): 318-322, 2007

ISSN 1549-3636

(C) 2007 Science Publications

\title{
Module Management Tool in Software Development Organizations
}

\author{
${ }^{1}$ Ahmad A. Al-Rababah and ${ }^{2}$ Mohammad A. Al-Rababah \\ ${ }^{1}$ Faculty of IT, Al-Ahliyyah Amman University, P.O.Box 252,19328, Amman, Jordan \\ ${ }^{2}$ Faculty of Science, Jerash Private University, Jordan
}

\begin{abstract}
Module management is an important task at developers' level in software development organizations. So we consider in this paper software as collection of several modules. Also we study a method of clustering of modules of a large software system. The need of doing such clustering is justified to be an important part of software management in the software development organizations. An algorithm of maintenance and modifications is presented for doing the clustering by the developers in the respective development organizations, whose complexity is calculated. The method will be very much helpful, as an essential tool, at the developer level in software development organizations for module management.
\end{abstract}

Key words: Link graphs, link matrix, MC, updated link matrix, coupling matrix

\section{INTRODUCTION}

The software architect devotes best effort for deriving an overall structural model of the system as a collection of several modules ${ }^{[1]}$, as far as possible by retaining loose links. This helps the developers to modify the modules almost independently, irrespective of the codes of other modules. But in reality, it is not always feasible to develop any system as a collection of only mutually independent modules. Module management ${ }^{[2-4]}$ is a very important job at the developers surface to improve the process visibility and the overall cost effectiveness. In object oriented model, modules are objects with private state and defined operations on that states. In the data flow models, modules are functional transformations. In both cases, module may be implemented as sequential components or as processes. An object-oriented model of a system architecture structures the system into a set of loosely coupled objects with well-defined interface ${ }^{[5,6]}$.

Modules and link graphs: Among these modules, a pair of modules may be mutually independent (ideal situation), or may have link. If a flow of data (or a flow of control) exists between these two modules, we say that they are linked, they are not independent. Thus, there are three types of link possible to be existing between a pair of modules:

* mo link, (if the modules are mutually independent)

* one link, (if there exists a flow of data or control from any one module to other, but not both ways).
* two links (if there exists a flow of data or control both ways).

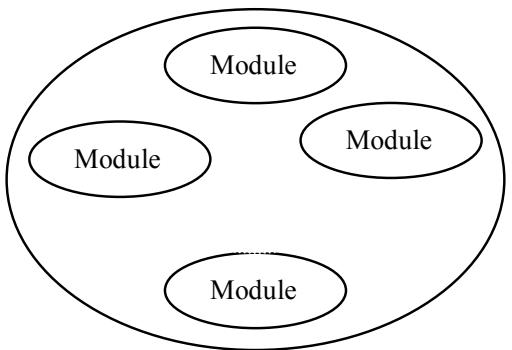

Fig. 1: Software

We ignore the trivial case of the existence of link of one module with itself. Thus, between a pair of modules, there could be at most two links (according to our concept of link as stated above). Consider a system $\mathrm{S}$ constituted of $\mathrm{n}$ modules developed under object oriented paradigm. Suppose that the modules are numbered from 1 to $\mathrm{n}$. Consider two modules module- $\mathrm{i}$ and module-j.

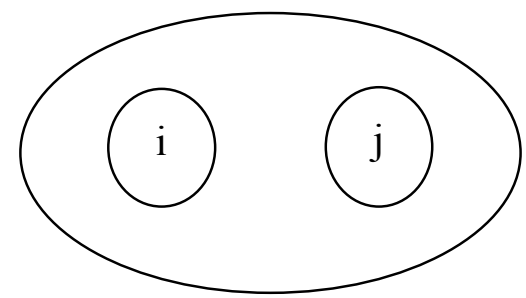

Fig. 2: No link graph between two modules

Corresponding Author: Ahmad A. Al-Rababah, Faculty of IT, Al-Ahliyyah Amman University, P.O. Box 252,19328, Amman, Jordan 


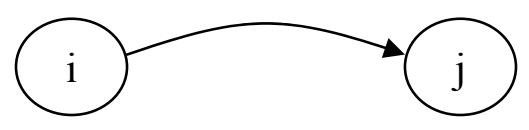

Fig. 3: and

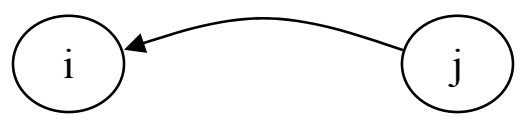

Fig.4: One-link graphs (directed)

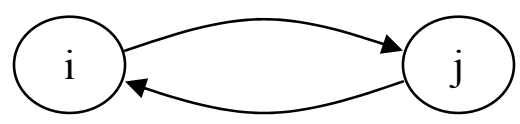

Fig. 5: Two-link graph (directed)

Let us assume that any two modules are linked with some weight $\mathrm{W}$ (number of links) such that $\mathrm{W}_{\mathrm{ij}}=$ weight of the link between module-i and module-j

Then,

$$
\begin{aligned}
\mathrm{W}_{\mathrm{ij}} & =0, \text { if no link } \\
& =1, \text { if one link } \\
& =2, \text { if two links. }
\end{aligned}
$$

Clearly, $W_{i j}$ is commutative with respect to its indices $i$ and j. i.e. $\mathrm{W}_{\mathrm{ij}}=\mathrm{W}_{\mathrm{ji}}$.

Then the link graph of the system $\mathrm{S}$ will be an undirected graph as shown bellow:

For $\mathrm{n}=1$

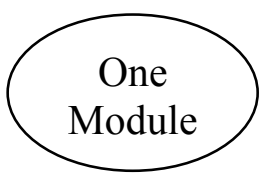

Fig. 6: S

For $\mathrm{n}=2$

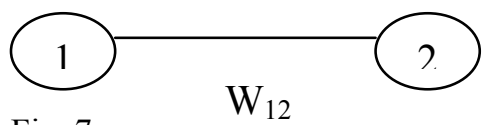

Fig. 7:

For $\mathrm{n}=3$

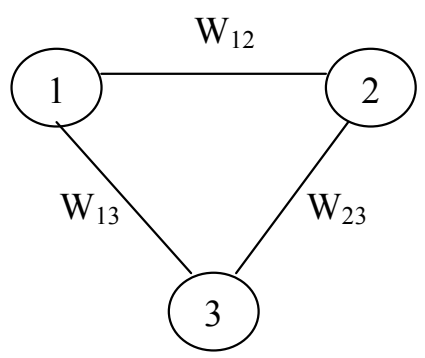

Fig. 8: W
For $\mathrm{n}=4$

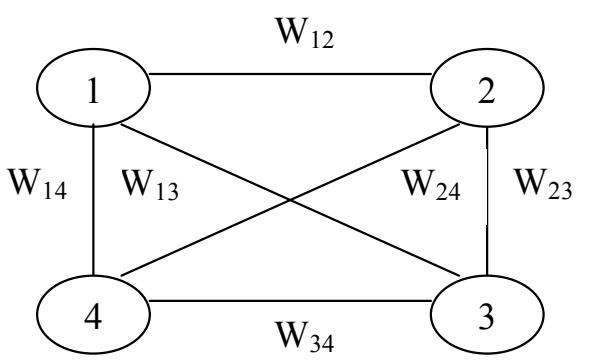

Fig. 9: and so on.

Link matrix and the integer set Zr: Now, let us divide each element $\mathrm{W}_{\mathrm{ij}}$ into two parts such that

* $\quad \mathrm{W}_{\mathrm{ij}}=\mathrm{l}_{\mathrm{ij}}+\mathrm{l}_{\mathrm{ji}}$,

* $\mathrm{l}_{\mathrm{ij}}=1$, if module- $\mathrm{i}$ has to send a deliverable to module-j

$=0$, if module-i will not have to send any deliverable to module-j. (and same type of definition is also true for every $l_{\mathrm{ij}}, \mathrm{i} \neq \mathrm{j}$ ).

We assume that $\mathrm{l}_{\mathrm{ii}}=D$ (don't care symbol), (or the number 0) ignoring theorases of deliverables which are internal only. Obviously, $\mathrm{m}_{\mathrm{ij}}$ and $\mathrm{m}_{\mathrm{ji}}$ are not necessarily equal. They are one way link values only. Then the Link matrix of the system $\mathrm{S}$ is defined as the square matrix $\mathrm{L}=\left[\mathrm{l}_{\mathrm{ij}}\right]$

In the link matrix L, each row could be viewed as the modules to perform the role of suppliers and each column could be viewed as modules to perform the role of consumers.

Example of a link matrix: Consider a system $\mathrm{S}$ with 4 modules 1,2, 3 and 4

$$
L=\frac{1}{1}\left[\begin{array}{llll}
0 & 1 & 3 & 4 \\
3 \\
4
\end{array}\left[\begin{array}{llll}
0 & 0 & 1 & 0 \\
1 & 0 & 0 & 1 \\
0 & 0 & 1 & 0
\end{array}\right]\right.
$$

In this link matrix, the data $l_{23}=1$ means that the module- 2 will send a deliverable to module- 3 , whereas the data $1_{32}=0$ means that module- 3 will not. The data $1_{14}=0$ and $1_{41}=0$ combined signifies that module -1 and module- 4 are mutually independent, because $\mathrm{W}_{14}$ will be zero in this case. If numbers of 1's are more in the matrix L, it implies that there are many modules linked with each other. The ideal situation under object oriented paradigm is that the number of 0 's in nondiagonal positions of $\mathrm{L}$ should be more and more. 


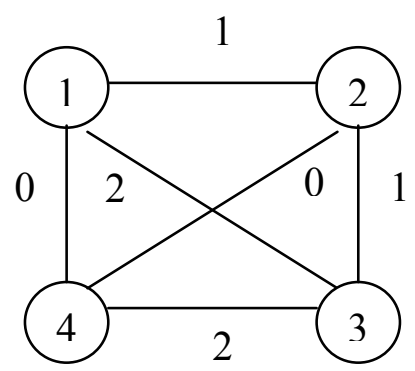

Fig. 10: Link-graph of S (Undirected)

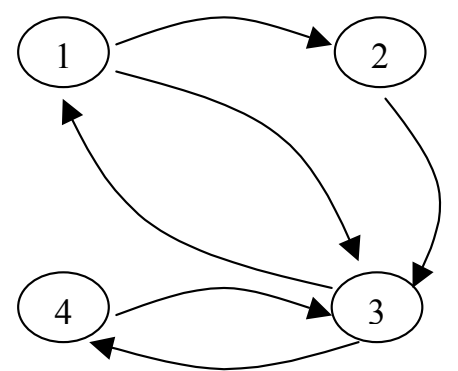

Fig. 11: Link-graph of S (Directed)

Integer-Set $\mathbf{Z}_{\mathrm{r} \text { : Let }} r \in \stackrel{+}{I}$. We define integer-set $\mathrm{Z}_{\mathrm{r}}$ as the set of all non negative integer less than or equal to $r$. Thus, $Z_{2}=\{0,1,2\}, Z_{5}=\{0,1,2,3,4,5\}$ etc.

Clustering of modules: By grouping together the modules which interact with each other very closely, it's certainly possible to reduce the efforts in the software maintenance ${ }^{[3,7]}$. Two or more closely related modules, if grouped as one object, forms a modulecluster (MC).

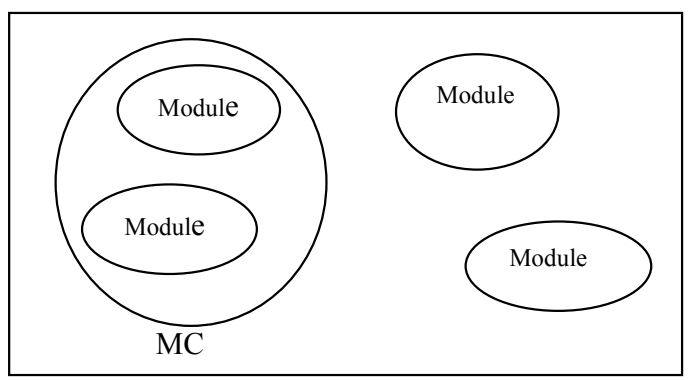

Fig. 12: Software system

Now, we study how to group two modules to form a cluster and then how to update the link-matrix of the system after completing each clustering. Consider the system $\mathrm{S}$ as chosen in example earlier.

Suppose that we like to group module-1 and module-2 to form a new cluster MC.

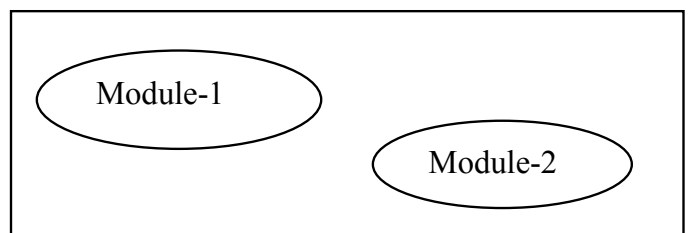

Fig. 13: One Cluster $\mathrm{C}_{1}$

For this we consider the sub matrix $\mathrm{L}_{1}$ of $\mathrm{L}$ corresponding to row-1, row-2 and column-1, column-2 given by

$L_{l}=1\left[\begin{array}{cc}1 & 2 \\ 2 & 1 \\ 0 & 0\end{array}\right]$

Now this new cluster $C_{1}$ is to be treated as good as one bigger module, say module- 1 (specified by bold one). Thus, at present the system $\mathrm{S}$ consists of one cluster (the new created module-1), module- 3 and module- 4 . The total number of deliverables from this cluster to module-i, (for $i=2,3$ ) is clearly equal to the sum of the numbers of deliverables from module- 1 and module-2 to module-i. The link values will now be changed accordingly, and thus our updated link-matrix for the system $\mathrm{S}$ will be as below:

$$
L=1\left[\begin{array}{lll}
1 & 2 & 3 \\
2 & 2 & 0 \\
1 & 0 & 1 \\
0 & 1 & 0
\end{array}\right]
$$

It's to be noticed that in this updated link matrix L, $1_{11}$ is not zero but 1 , which is clear from the submatrix $\mathrm{L}_{1}$, and the updated link matrix $\mathrm{L}$ is still a square matrix. Also in the initial link matrix $L$ of the system $S$, the matrix-elements $\in Z_{1}$. In the first updated link matrix $L$, the elements $\in Z_{2}$. We will see next that in the $\mathrm{p}_{\text {th }}$ updated link matrix $\mathrm{L}$ of the system $\mathrm{S}$, the elements $\in Z_{\mathrm{p}+\mathrm{k}}$ where $\mathrm{k} \geq 1$. Thus, after this clustering, the software system $\mathrm{S}$ will be apparently as shown in Fig. 13 to the developers' eyes. The method could be generalized to group together two clusters to form a bigger cluster of clusters. For example, the following two figures can be seen:

* New cluster (by grouping one cluster and one module)

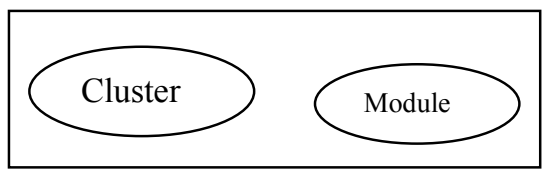

Fig. 14: Cluster 
* New cluster (by grouping two clusters)

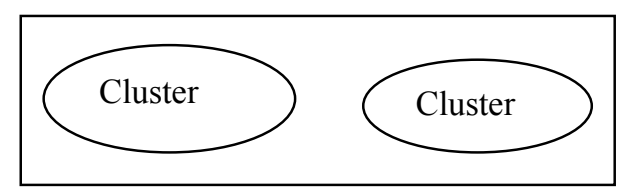

Fig. 15: Cluster

Couplingmatrix M: Each module could be viewed as a trivial cluster initially. However, if we consider two clusters $\mathrm{C}_{\mathrm{r}}$ and $\mathrm{C}_{\mathrm{s}}$, then these are two parameters which we shall consider now. The parameters are:

* maximum number $(\mathrm{m})$ of possible links between $\mathrm{C}_{\mathrm{r}}$ and $\mathrm{C}_{\mathrm{s}}$

* actual number (a) of links existing between $\mathrm{C}_{\mathrm{r}}$ and $\mathrm{C}_{\mathrm{s}}$

Clearly, the ratio $\left(\frac{a}{m}\right)$ will be a member in the closed interval $[0,1]$. This number (collectively for every pair of clusters) constitutes the coupling matrix $\mathrm{M}$ of a system S. For example, at the initial stage of the system $\mathrm{S}$ when each cluster contains exactly one module, the number $\mathrm{a} \in \mathrm{Z}_{2}$ and $\mathrm{m}=2$.

Thus, $\left(\frac{a}{m}\right) \in\{0,1 / 2,1\}$. The elements of the coupling matrix $\mathrm{MC}=\left[\mathrm{m}_{\mathrm{ij}}\right]$ are defined now as below. (There are three different cases):

Case 1: Suppose that both of the clusters contains single module only.

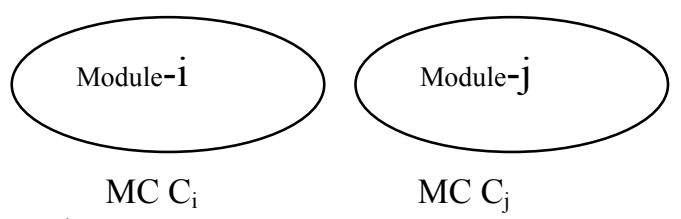

Fig. 16:

In this case, $\mathrm{a}=\mathrm{w}_{\mathrm{ij}}=\mathrm{1}_{\mathrm{ij}}+\mathrm{l}_{\mathrm{ji}}$ and $\mathrm{m}=2$ where $\mathrm{1}_{\mathrm{ij}}, 1_{\mathrm{ji}}$ are elements of the current link matrix $L$ of the system S.

Thus, $m_{i j}=\frac{w_{i j}}{2}=\frac{l_{i j}+l_{j i}}{2}$ Clearly, $\mathrm{m}_{\mathrm{ij}} \in[0,1]$.

Case 2: Suppose that a cluster $C_{i}$ contains $n_{i}$ number of modules and another cluster $C_{j}$ contains only one module module-j.

In this case, $\mathrm{m}_{\mathrm{ij}}=\frac{l_{i j}+l_{j i}}{2 n_{i}}$, because $\mathrm{m}=2 \mathrm{n}_{\mathrm{i}}$ here.

Clearly $\mathrm{m}_{\mathrm{ij}} \in[0,1]$.
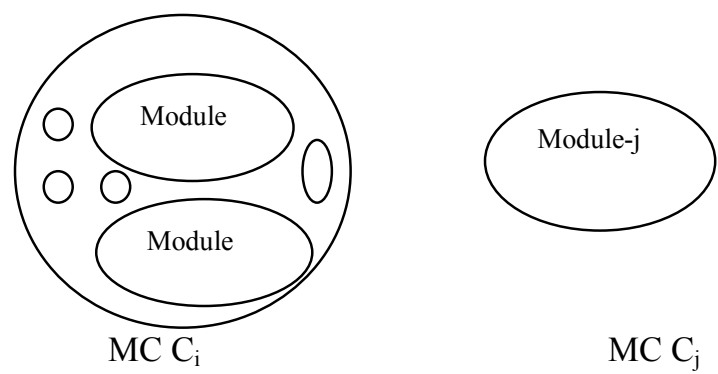

Fig. 17: Modules

Case 3: Suppose that a cluster $C_{i}$ contains $n_{i}$ number of modules and another cluster $C_{j}$ contains $n_{j}$ number of modules.
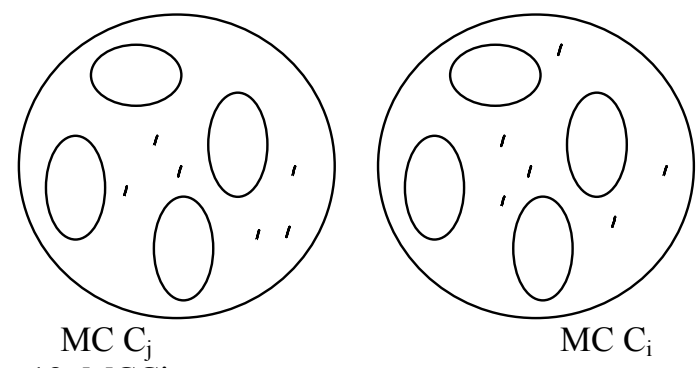

Fig. 18: MCCi

In this case, $\mathrm{m}=2 \mathrm{n}_{\mathrm{i}} \mathrm{n}_{\mathrm{j}}$, and $\mathrm{m}_{\mathrm{ij}}=\frac{l_{i j}+l_{j i}}{2 n_{i} n_{j}}$,

Where $l_{\mathrm{ij}}$ and $\mathrm{l}_{\mathrm{ji}}$ are the elements of the last updated link matrix of the software system S. In this case also, $\mathrm{m}_{\mathrm{ij}} \in[0,1]$. Clearly, the coupling matrix $\mathrm{M}$ is a square and a symmetric matrix. If $\mathrm{m}_{\mathrm{ij}}=0$, it indicates that the two clusters $C_{i}$ and $C_{j}$ are mutually independent. If $m_{i j}$ is a low value, it indicates that the two clusters $C_{i}$ and $\mathrm{C}_{\mathrm{j}}$ are loosely coupled and if it's a high value (close to 1) then it indicates that the two clusters are strongly coupling. At every stage we shall look at the coupling matrix $\mathrm{M}$ to watch which pair of clusters is most strongly coupling and in the next step we will group these two clusters to form a new cluster. This is the main paradigm of clustering in our work here. Such pair of clusters is called Strongly Coupled Clusters (SCC).

The task of clustering by this method is continued until we reach at a single cluster (which is equivalent to the complete software system S). But it's most preferable to stop once a small number of meaningful and low-coupling clusters have been reached upon.The final number of clusters should be optimal in the context of maintenance and modifications to be done by the developers in the respective development organizations. We shall not cluster $\mathrm{C}_{\mathrm{i}}$ and $\mathrm{C}_{\mathrm{j}}$ into one cluster if $\mathrm{m}_{\mathrm{ij}}=0$, or very close to zero. For this the Developers (or the Software Development 
Organization) choose a threshold value $\mu$ which is very close to zero. Now, we present an algorithm for the above task of clustering.

Algorithm for module clustering: Here, we present an algorithm for module clustering which is an improved version of the existing algorithms ${ }^{[7]}$. At each iteration in this algorithm, two clusters with strongest couplingvalue are merged to form a new cluster and the link matrix is updated immediately. Since the coupling matrix $M$ is a symmetric matrix, the elements of its uppers triangular region are sufficient for us to consider. The algorithm uses an important data structure which is an array $\mathrm{N}=<\mathrm{N}_{1}, \mathrm{~N}_{2}, \ldots, \mathrm{N}_{\mathrm{n}}>$ of $\mathrm{n}$ elements, where $\mathrm{N}_{\mathrm{i}}$ is number of modules present in the $i_{\text {th }}$ cluster.

Clearly, at the initial stage, $\mathrm{N}_{\mathrm{i}}=1, \forall \mathrm{i}=1,2,3, \ldots \ldots, \mathrm{n}$.

Module-clustering Algorithm

Step 1: Calculate the initial link matrix $L$ and the upper triangular portion of the coupling matrix $\mathrm{M}$, (i.e. $\forall \mathrm{i}$ and $\forall$ j where $1 \leq \mathrm{i} \leq \mathrm{j}$ $\leq \mathrm{n})$

Step 2: Repeat step-3 through step-6, (n-1) times.

Step 3: Find the pair of SCC with coupling value more than $\mu$. (if more than one pair exists, chose any one arbitrarily) which are $\mathrm{C}_{\mathrm{i}}$ ' and $\mathrm{C}_{\mathrm{j}}{ }^{\prime}$

where $1 \leq \mathrm{i}^{\prime} \leq \mathrm{j}^{\prime} \leq \mathrm{n}$ (this inequality which can be assumed with no loss of generality). Calculate $\mathrm{m}_{\mathrm{i}^{\prime} \mathrm{j}}{ }^{\prime}=\max \left\{\mathrm{m}_{\mathrm{ij}}\right\}, 1 \leq$ $\mathrm{i} \leq \mathrm{j} \leq \mathrm{n}$

Step 4: Merge $C_{i}{ }^{\prime}$ and $C_{j}$ ' to form a new cluster $C_{i}$.

Step 4.1: $\mathrm{N}_{\mathrm{i}}, \leftarrow \mathrm{N}_{\mathrm{i}},+\mathrm{N}_{\mathrm{j}}$,

Step 4.2: Update $i_{\text {th }}$ column ( $i_{\text {th }}$ row ) of the link matrix such that

$\forall \mathrm{k}=1,2, \ldots \mathrm{n}$

where, $\mathrm{k} \neq \mathrm{i}$, j'

$1_{i^{\prime} \mathrm{k}} \leftarrow 1_{i^{\prime} \mathrm{k}}+1_{\mathrm{ik}}$

$\mathrm{l}_{\mathrm{i}^{\prime} \mathrm{k}} \leftarrow 0$

Step 5: $\quad$ Ignore now the cluster $\mathrm{j}$ ', and set $\mathrm{N}_{\mathrm{ji}} \leftarrow 0$.

Step 6: Update the coupling matrix M.

Step 6.1: Update the $i_{\text {'th }}$ row of $M$ by doing:

$\forall \mathrm{k}=1,2, \ldots \mathrm{n}$

where $\mathrm{k} \neq \mathrm{i}$

$\mathrm{m}_{\mathrm{kj}} \leftarrow \mathrm{m}_{\mathrm{ki}}$

Step 6.2: Update $i$ 't column of $M$ by doing:

$\forall \mathrm{k}=1,2, \ldots \mathrm{n}$

where $\mathrm{k} \neq \mathrm{i}$

$\mathrm{m}_{\mathrm{j}^{\prime} \mathrm{k}} \leftarrow \mathrm{m}_{\mathrm{i}^{\prime} \mathrm{k}}$

Step 7: Stop.

Complexity of the algorithm: To calculate the complexity $T(n)$ of the module clustering algorithm we see that:
In step-1, there will be computation of ${ }^{\mathrm{n}} \mathrm{C}_{2}$ elements of the coupling matrix $\mathrm{M}$. The complexity of this step is $\mathrm{O}\left(\mathrm{n}^{2}\right)$

Step-3 involves a sorting algorithm, in worst-case which will consume $\mathrm{O}\left(\mathrm{n}^{2}\right)$ amount of time (in case the developers seeks alternative SCC from the sorted clusters). All other steps from 4 to 6 have the complexity $\mathrm{O}(\mathrm{n})$ respectively. Thus, the portion from step-3 through step-6 has the complexity $=\mathrm{O}\left(\mathrm{n}^{2}\right)+$ $\mathrm{O}(\mathrm{n})=\mathrm{O}\left(\mathrm{n}^{2}\right)$.

But, due to the repetition of this computation for (n-1) times ( because of step 2), we have the total complexity of the algorithm given by

$\begin{array}{rll}\mathrm{T}(\mathrm{n}) & = & \mathrm{O}\left(\mathrm{n}^{2}\right)+(\mathrm{n}-1) * \mathrm{O}\left(\mathrm{n}^{2}\right) \\ & = & \mathrm{O}\left(\mathrm{n}^{3}\right)\end{array}$

Thus, we see that the algorithm is a $P$-class algorithm.

\section{CONCLUSION}

We have studied here a methodology for module clustering in a huge type of software system which has been developed with a large number of modules. The need of doing such clustering is justified to be an important part of software management in the software development organizations. We present a polynomial time algorithm for module clustering and the method can be well applicable especially for the large software systems developed with object oriented approach. The final number of clusters should be optimal in the context of maintenance and modifications to be done by the developers in the respective development organizations.

\section{REFERENCES}

1. Gao, J.Z. and H.S.J. Tsao, 2006. Testing and Quality Assurance for Component-Based Software. Artech House Publishers.

2. Berczuk, S. and B. Appleton, 2002. Software Configuration Management Patterns: Effective Teamwork. Addison-Wesley.

3. Pullum, L.L., 2001. Software Fault Tolerance Techniques and Implementation, Norwood, MA: Artech House.

4. Summerville, I., 2002. Software Engineering, Pearson Education Asia. New Delhi.

5. Kartashev, S., 1990. Supercomputing Systems: Architectures, Design and Performance. New York.

6. Zedan, H.S.M. and A. Cau, 1999. Object-Oriented Technology and Computing Systems ReEngineering. Horwood Publishing Limited.

7. Councill, W. and G.T. Heineman, 2003. Definition of a Software Component and its Elements. Addison-Wesley. 